\title{
El ingreso de las músicas colombianas a la educación superior del Conservatorio
} del Tolima

Introduction of colombian's musical styles in higher education at the Tolima Conservatory

Boris Alfonso Salinas Arias boris.salinas@conservatoriodeltolima.edu.co

Conservatorio del Tolima

Tolima, Colombia

ORCID: https://orcid.org/0000-0002-2439-744X

doi: 10.7203/LEEME.41.10220

Recibido: 20-07-2017 Aceptado: 01-03-2018. Contacto y correspondencia: Boris Alfonso Salinas Arias Conservatorio del Tolima, C/ 9, 1-18, Ibagué (Tolima), Colombia.

\section{Resumen}

La Educación musical en el Conservatorio del Tolima se ha enfocado en la Música Clásica o Culta Europea, fenómeno que no es aislado sino común en un país que tradicionalmente ha dejado por fuera de la academia a las Músicas Colombianas. No obstante, en el año 2011 estas músicas ingresaron de forma decidida al Currículo del programa Licenciatura en Música. El presente trabajo es un análisis curricular que establece las razones por las cuales las Músicas Colombianas ingresan a la Formación profesional de esta institución, entre las que se encuentra la visión del Estado Colombiano sobre la Música como herramienta de pacificación en medio del Conflicto Armado, la creación de un Plan Nacional de Música guiado hacia la Convivencia y apoyado en sus orígenes por la política de intervención militar de Estados Unidos llamada Plan Colombia. Asimismo, se discute la adaptación que hizo la Institución a su programa académico de la política Nacional de Guerra.

Palabras clave: Músicas Colombianas, Análisis Curricular, Educación Musical, Conflicto Armado.

\section{Abstract}

Music education in the Conservatory of Tolima has focused on Classical Music or European art music, a phenomenon that is not isolated but instead common in a country that has traditionally Colombian music out of the academy. However, in 2011, these musical styles became part of the curriculum of the Bachelor of Music program. The present work is a curricular analysis and establishes the reasons why Colombian Music began to form part of the Professional Formation of this institution, among these reasons the Colombian State's vision of music as a tool of pacification in the Armed Conflict and the creation of a National Music Plan to establish coexistence, originally supported by the United States military intervention policy called Plan Colombia. Also discussed is the adaptation made by the Institution to its National War Policy academic program.

Key words: Colombian Music, Curricular Analysis, Music Education, Armed Conflict.

(c) BORIS ALFONSO SALINAS ARIAS. THE CONTENT OF THIS ARTICLE IS THE SOLE RESPONSIBILITY OF THE AUTHORS. THE REVISTA ELECTRÓNICA DE LEEME AND UNIVERSITAT DE VALĖNCIA ARE NOT LIABLE FOR ANY LEGAL ACTIONS THAT MAYARISE INVOLVING THE ARTICLE'S CONTENT. REVISTA ELECTRÓNICA DE LEEME -LISTA ELECTRÓNICA EUROPEA DE MÚSICA EN LA EDUCACIÓN-HTTP://MUSICA.REDIRIS.ES.ISSN: 1575-9563 EDITORES: UNIVERSIDAD DE VALENCIA Y JESÚS TEJADA GIMÉNEZ. VISIBILIDAD DE ESTA REVISTA: EBSCO, CINDOC RESH, REDIB, RILMCOREJOURNALS, SUDOC, ULRICHS, ESTA REVISTA ESTA PUBLICADA CON EL APOYO INSTITUCIONAL DE REDIRIS-CONSEJO SUPERIOR DE INVESTIGACIONES CIENTIFICAS Y ES DE ACCESO LIBRE.CREATIVECOMMONSLICENSE 4.O BY 


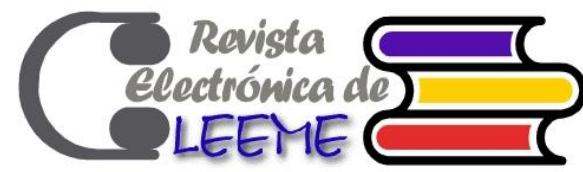

\section{Introducción}

El Conservatorio del Tolima fue creado en 1906 como proyecto educativo musical no formal, que pasó a ser un Bachillerato Musical en 1959, ofertando un programa de educación media en el que los estudiantes se graduaban como instrumentistas sinfónicos en un periodo de ocho años. Desde 1980 este Conservatorio se convirtió en Institución de Educación Superior empero, comenzó a funcionar como tal hasta 1994 cuando ofertó su primer programa profesional, la Licenciatura en Música (Resolución $N^{o} 645$ del ICFES).

Desde su nacimiento hasta hoy la Licenciatura en Música ha tenido seis versiones curriculares siendo la más reciente la del año 2017. Sin embargo, fue la versión número 5 del año 2011 la que incorporó de manera estructural a las Músicas Colombianas en su currículo. Este hecho no es menor pues de manera tradicional la educación musical profesional no solo en la ciudad de Ibagué sino en Colombia se ha basado en el estudio de la Música Europea relegando a un segundo plano a las Músicas Colombianas, fenómeno que ha sido documentado por autores como Ochoa (2011) y Ayala (2013).

El objetivo central de este documento es analizar y comprender por qué, luego de tener una escasa presencia, ingresó la música colombiana al currículo del programa Licenciatura en Música del Conservatorio del Tolima, así como identificar las fuerzas e intereses que posibilitaron ese cambio, entre los cuales se encuentra el Conflicto Armado Colombiano (de 1948 a hoy) y el discurso utilitario sobre la Música.

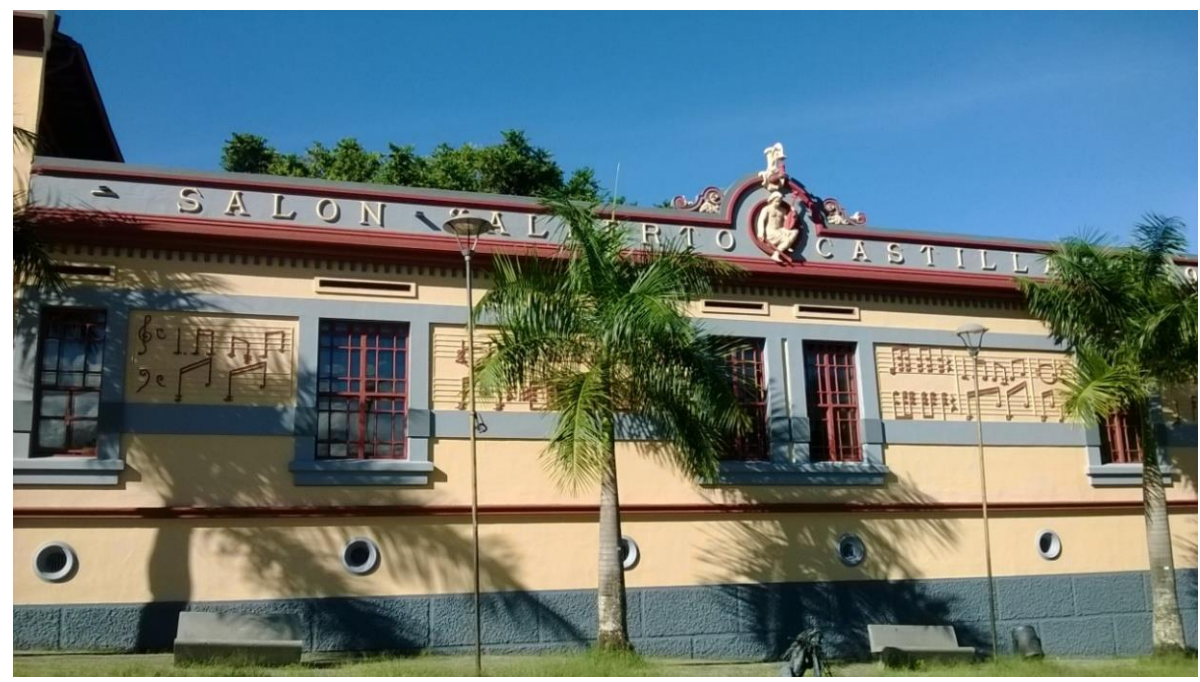

Figura 1. Sede tradicional del Conservatorio del Tolima, Salón Alberto Castilla, edificio declarado Monumento Nacional de Colombia, Ley 112 de 1994. Fotografía tomada por el Autor, Ibagué, Conservatorio del Tolima, 2017.

(c) BORIS ALFONSO SALINAS ARIAS. THE CONTENT OF THIS ARTICLE IS THE SOLE RESPONSIBILITY OF THE AUTHORS. THE REVISTA ELECTRÓNICA DE LEEME AND UNIVERSITAT DE VALÈNCIA ARE NOT LIABLE FOR ANY LEGAL ACTIONS THAT MAYARISE INVOLVING THE ARTICLE'S CONTENT. REVISTA ELECTRÓNICA DE LEEME - LISTA ELECTRÓNICA EUROPEA DE MÚSICA EN LA EDUCACIÓN-HTTP://MUSICA.REDIRIS.ES.ISSN: 1575-9563 EDITORES: UNIVERSIDAD DE VALENCIA Y JESÚS TEJADA GIMÉNEZ. VISIBILIDAD DE ESTA REVISTA: EBSCO, CINDOC (CESIC), CITEFACTOR, COPAC, DIALNET, DICE (CSIC), DOAJ, E-REVISTAS (CSIC), EBSCO PREMIER, ERIH+, GALECENGAGELEARNING, IN-RECS, IRESIE, LATINDEX, MIAR, OCLCWORLDCAT, RESH, REDIB, RILMCOREJOURNALS, SUDOC, ULRICHS, ESTA REVISTA ESTÁ PUBLICADA CON EL APOYO INSTITUCIONAL DE REDIRIS-CONSEJO SUPERIOR DE INVESTIGACIONES CIENTIFICAS Y ES DE ACCESO LIBRE.CREATIVECOMMONSLICENSE 4.0 BY 


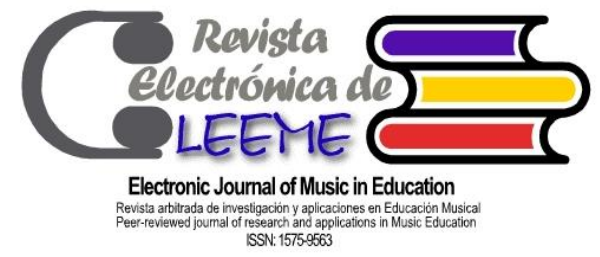

EL INGRESO DE LAS MÚSICAS COLOMBIANAS BORIS ALFONSO SALINAS ARIAS CONSERVATORIO DEL TOLIMA (REVISTAELECTRÓNICA DE LEEME) NÚMERO 41, PP. 1-15 HTTPS://OJS.UV.ES/INDEX.PHP/LEEME/INDEX

\title{
2. Estado de la Cuestión
}

Aunque la inclusión de las Músicas Colombianas en el Conservatorio del Tolima es un cambio nuevo y sustancial en un país que, tradicionalmente, formó académicamente a sus músicos para interpretar música Clásica, los antecedentes que demuestran esfuerzos para lograr esa inclusión llegan desde los años 90 con la creación de la Licenciatura. El documento El papel de la música popular en la Facultad de Educación y Artes del Conservatorio del Tolima contextualiza este panorama:

\begin{abstract}
"Tradicionalmente en el Conservatorio del Tolima se han privilegiado las llamadas "músicas cultas", como fuente principal de enseñanza y de interpretación musical. Era no sólo mal visto sino cohibida la inclinación espontánea de estudiantes y docentes de recrear aires musicales populares... Esta es la historia de la celosa tradición musical (de corte clásico y origen europeo) que se impone en la institución, haciendo negación y prohibición de la música popular, llegando a penalizar a quienes se arriesgaban a interpretarla con castigos que iban desde las amonestaciones verbales hasta quitar el instrumento por varios días, dejarlos en el colegio después de la salida (de cinco a seis de la tarde) o, escribir frases en un cuaderno: "no debo tocar música popular" (Camacho, Díaz y Pérez, 2000, p.1-2).
\end{abstract}

Este documento explica que con la creación de la Licenciatura en Música se estaba explorando un nuevo camino para la institución en el que la incorporación de las Músicas Colombianas dejó de ser un tema tabú. Este fenómeno fue impulsado por eventos como la crisis económica institucional que impidió continuar con la vinculación de profesores extranjeros, lo que llevó a la contratación de docentes graduados de la institución quienes "se debaten entre esta historia de negación y de osadía estudiantil: son ellos mismos portadores de esta ambigüedad musical entre lo clásico y popular" (Camacho et al., 2000, p.3). Asimismo, entre los sucesos que influyeron para hacer posible un cambio de pensamiento se encuentra el quinto centenario del descubrimiento de América el cual propició un "nuevo ambiente ideológico frente al reconocimiento y la reivindicación de las identidades locales y étnicas" (Camacho et al., 2000, p.3). A esto se sumó el que los intereses de los estudiantes de Licenciatura en Música fuese la Música popular, así como la necesidad institucional de buscar nuevas metodologías de enseñanza, "puesto que la tendencia del conservatorio era la educación clásica instrumental y no pedagógica" (p.4), sumado a que el Conservatorio no poseía experiencia en la formación de músicos profesionales ni de docentes de música. El programa de Licenciatura en Música hacía presión al cambio e inclusión de las Músicas Colombianas, mientras los otros programas tradicionales de formación seguían vigentes.

$\mathrm{Al}$ hacer una revisión documental de los micro currículos que existen en archivo del año 1996 al 2006 -no existen anteriores-, es visible que efectivamente se incluyeron diversas materias que poseían una iniciativa de reconocimiento y estudio de la cultura colombiana y Popular, incluyendo su Música, aunque con una visión euro céntrica. Asignaturas como Sociedades

(c) BORIS ALFONSO SALINAS ARIAS. THE CONTENT OF THIS ARTICLE IS THE SOLE RESPONSIBILITY OF THE AUTHORS. THE REVISTA ELECTRÓNICA DE LEEME AND UNIVERSITAT DE VALĖNCIA ARE NOT LIABLE FOR ANY LEGAL ACTIONS THAT MAYARISE INVOLVING THE ARTICLE'S CONTENT. REVISTA ELECTRÓNICA DE LEEME - LISTA ELECTRÓNICA EUROPEA DE MÚSICA EN LA EDUCACIÓN-HTTP://MUSICA.REDIRIS.ES.ISSN: 1575-9563 EDITORES: UNIVERSIDAD DE VALENCIA Y JESÚS TEJADA GIMÉNEZ. VISIBILIDAD DE ESTA REVISTA: EBSCO, CINDOC (CESIC), CITEFACTOR, COPAC, DIALNET, DICE (CSIC), DOAJ, E-REVISTAS (CSIC), EBSCO PREMIER, ERIH+, GALECENGAGELEARNING, IN-RECS, IRESIE, LATINDEX, MIAR, OCLCWORLDCAT, RESH, REDIB, RILMCOREJOURNALS, SUDOC, ULRICHS, ESTA REVISTA ESTÁ PUBLICADA CON EL APOYO INSTITUCIONAL DE REDIRIS-CONSEJO SUPERIOR DE INVESTIGACIONES CIENTIFICAS Y ES DE ACCESO LIBRE.CREATIVECOMMONSLICENSE 4.O BY 


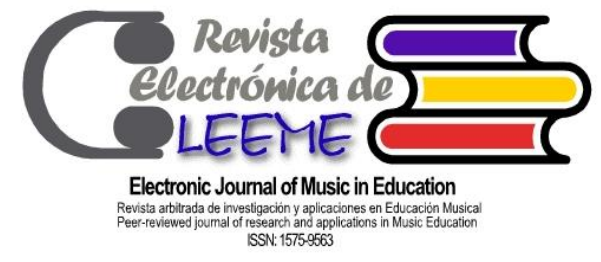

EL INGRESO DE LAS MÚSICAS COLOMBIANAS BORIS ALFONSO SALINAS ARIAS CONSERVATORIO DEL TOLIMA (REVISTAELECTRÓNICA DE LEEME) NÚMERO 41, PP. 1-15 $\frac{\text { HTTPS://OJS.UV.ES/INDEX.PHP/LEEME/INDEX }}{\text { DOI: } 10.7203 / \text { LEEME.41.10220 }}$

Tribales, Cultura Nacional y Regional y e Historia de la Música IV (Historia de la Música en Colombia), aparecen en el micro currículo desde el año 1996 (PAFE, Conservatorio del Tolima, 2006). De este periodo, se encuentra que los cambios de contenido y asignaturas coinciden con el cambio de docente. Evidentemente hay libertad de cátedra. No se puede decir que es prioridad de la institución fortalecer el estudio de las Músicas Colombianas, pero se permitía que los docentes la incluyeran en sus materias, se crearan ensambles e hicieran presentaciones artísticas.

Fue hasta el plan curricular del año 2011 de la Licenciatura en Música que se incluyó decididamente las Músicas Colombianas en el Pensum, a diferencia del pasado, donde en algunos casos el nombre mismo de la asignatura se dejaba entre paréntesis como Historia de la Música IV (Música Colombiana)- lo que denota una subordinación, visión lineal y euro céntrica de la Historia-. Se pasó de una inclusión tímida a una presencia notable. Entonces ¿Por qué ingresan decididamente y de manera estructural las Músicas Colombianas al programa de Licenciatura en Música del Conservatorio del Tolima?

\section{Método}

Este trabajo hace parte del proyecto de Investigación Las Músicas Colombianas en los programas de Formación Musical de Ibagué: apuntes sobre Currículo y Colonialidad, cuya estrategia central de investigación fue el Estudio de Caso (Páramo, 2013). El Caso escogido es el Conservatorio del Tolima, y el fenómeno a estudiar es la formación en Músicas Colombianas en sus programas de educación musical profesional, ante la paradoja que, siendo Ibagué la Ciudad Musical de Colombia, en su institución musical insignia no era permitida la Música Colombiana.

Para el presente escrito se configuró un análisis curricular partiendo del estudio de caso, que usa como fuentes de información los documentos -curriculares- del Conservatorio del Tolima, el Proyecto Educativo Institucional (PEI, 2015), Proyecto Educativo del Programa (PEP, 2010), así como los documentos Programas Académicos, Facultad de Educación y Artes, años 1997 2006. Legajos 1 al 19 del Conservatorio del Tolima (2006) -que en adelante llamaremos PAFE, y otros archivos a los cuales la investigación orientaba como los documentos del Consejo Nacional de Política Económica y Social (CONPES) del Estado Colombiano, entre otros.

\section{Resultados}

Al analizar el Proyecto Educativo del Programa (PEP) de Licenciatura en Música en la versión del 3 de agosto de 2010, se logra identificar que las razones por las cuales se modifica el programa obedecen a una mirada utilitaria de la música y la preocupación del Conservatorio del Tolima de garantizar un campo laboral a sus graduados.

(c) BORIS ALFONSO SALINAS ARIAS. THE CONTENT OF THIS ARTICLE IS THE SOLE RESPONSIBILITY OF THE AUTHORS. THE REVISTA ELECTRÓNICA DE LEEME AND UNIVERSITAT DE VALĖNCIA ARE NOT LIABLE FOR ANY LEGAL ACTIONS THAT MAYARISE INVOLVING THE ARTICLE'S CONTENT. REVISTA ELECTRÓNICA DE LEEME -LISTA ELECTRÓNICA EUROPEA DE MÚSICA EN LA EDUCACIÓN-HTTP://MUSICA.REDIRIS.ES.ISSN: 1575-9563 EDITORES: UNIVERSIDAD DE VALENCIA Y JESÚS TEJADA GIMÉNEZ. VISIBILIDAD DE ESTA REVISTA: EBSCO, CINDOC (CESIC), CITEFACTOR, COPAC, DIALNET, DICE (CSIC), DOAJ, EREVISTAS (CSIC), EBSCO PREMIER, ERIH+, GALECENGAGELEARNING, IN-RECS, IRESIE, LATINDEX, MIAR, OCLCWORLDCAT, RESH, REDIB, RILMCOREJOURNALS, SUDOC, ULRICHS, ESTA REVISTA ESTÁ PUBLICADA CON EL APOYO INSTITUCIONAL DE REDIRIS-CONSEJO SUPERIOR DE INVESTIGACIONES CIENTIFICAS Y ES DE ACCESO LIBRE.CREATIVECOMMONSLICENSE 4.O BY 


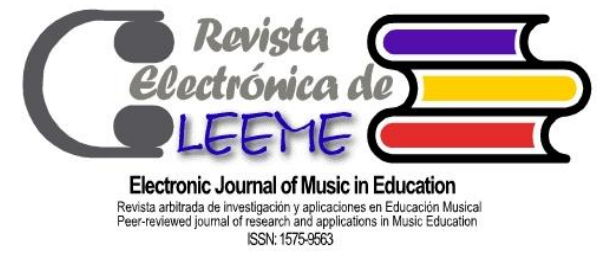

BORIS ALFONSO SALINAS ARIAS
EL INGRESO DE LAS MÚSICAS COLOMBIANAS A LA EDUCACIÓN SUPERIOR DEL CONSERVATORIO DEL TOLIMA (REVISTAELECTRÓNICA DE LEEME) NÚMERO 41, PP. 1-15 HTTPS://OJS.UV.ES/INDEX.PHP/LEEME/INDEX

En la justificación de la Licenciatura se encuentra desde el primer párrafo unas oportunidades potenciales o existentes de desempeño enmarcadas por las necesidades del país y la región en la visión del Gobierno Nacional y los estudios realizados por sus entes, el Ministerio de Educación y el Ministerio de Cultura:

\begin{abstract}
"Además de las acciones conducentes al fortalecimiento de la educación artística y en particular la musical en las instituciones de educación básica y media en Colombia, el gobierno nacional a través del Ministerio de Cultura ha formulado el Plan Nacional de Música para la Convivencia en el cual traza como política pública la formación musical como un derecho para todos” (PEP, 2010, p.1).
\end{abstract}

En el PEP (2010) de la Licenciatura se identifica como "Oportunidades potenciales o existentes de desempeño y tendencias del ejercicio profesional” (p.1), y define como relación entre "El programa y las necesidades el país y la región" (p.3), a dos políticas de Estado. La primera de ellas es la política de Educación Artística emanada desde la Ley General de Cultura Ley 397 de 1997- y estructurada en el Documento Orientaciones pedagógicas para la educación artística y cultural en la educación preescolar, básica y media del Ministerio de Educación (2008) en el cual se describe a nivel general la importancia de la música en la escuela. De allí la Licenciatura en Música toma algunos aspectos importantes para la configuración del programa como son la forma de elaborar las Competencias y el sentido de los espacios educativos, pero no da luces sobre las Músicas Colombianas, objeto del presente documento.

La segunda política Estatal en que se inspira la Licenciatura en Música es el Plan Nacional de Música para la Convivencia (PNMC), la cual orientó el diseño curricular de la versión 2011 del programa, y permitió -a la luz del estudio curricular- el ingreso estructural de las Músicas Colombianas al Conservatorio del Tolima, ¿Por qué ingresan las Músicas Colombianas al Currículo del programa Licenciatura en Música del Conservatorio del Tolima? La respuesta a esta pregunta está en la relación existente entre el PNMC y el Conservatorio del Tolima.

El programa Licenciatura en Música en su PEP (2010) poco habla de las Músicas Colombianas, no obstante, asume en su Currículo cuatro áreas de énfasis o profundización entre las cuales se encuentra la Licenciatura en Música con énfasis en conjuntos de música tradicional colombiana, ¿Qué relación posee esto con el PNMC? La propuesta musical del PNMC fija un completo paquete que abarca la dotación de instrumentos musicales e infraestructura, formación musical, investigación, creación, divulgación, emprendimiento, generación de empresas culturales, y publicación editorial; su visión pedagógica se basa en la práctica musical colectiva a partir de grupos y ensambles en los que se escogió crear o fortalecer cuatro tipo de agrupaciones musicales (i) Música popular tradicional, (ii) las Bandas -sinfónicas-, (iii) Coros, y (iv) Orquestas sinfónicas. Estas agrupaciones fueron escogidas por PNMC que realizó un diagnóstico sobre la actividad de este tipo de agrupaciones en el país:

(c) BORIS ALFONSO SALINAS ARIAS. THE CONTENT OF THIS ARTICLE IS THE SOLE RESPONSIBILITY OF THE AUTHORS. THE REVISTA ELECTRÓNICA DE LEEME AND UNIVERSITAT DE VALĖNCIA ARE NOT LIABLE FOR ANY LEGAL ACTIONS THAT MAYARISE INVOLVING THE ARTICLE'S CONTENT. REVISTA ELECTRÓNICA DE LEEME -LISTA ELECTRÓNICA EUROPEA DE MÚSICA EN LA EDUCACIÓN-HTTP://MUSICA.REDIRIS.ES.ISSN: 1575-9563 EDITORES: UNIVERSIDAD DE VALENCIA Y JESÚS TEJADA GIMÉNEZ. VISIBILIDAD DE ESTA REVISTA: EBSCO, CINDOC (CESIC), CITEFACTOR, COPAC, DIALNET, DICE (CSIC), DOAJ, EREVISTAS (CSIC), EBSCO PREMIER, ERIH+, GALECENGAGELEARNING, IN-RECS, IRESIE, LATINDEX, MIAR, OCLCWORLDCAT, RESH, REDIB, RILMCOREJOURNALS, SUDOC, ULRICHS, ESTA REVISTA
CIENTIFICAS Y ES DE ACCESO LIBRE.CREATIVECOMMONSLICENSE 4.0 BY 


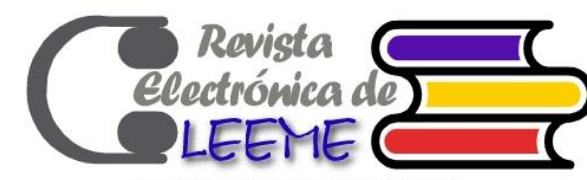

Electronic Journal of Music in Education

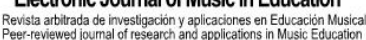

of research and applicain
ISSN: 15759563

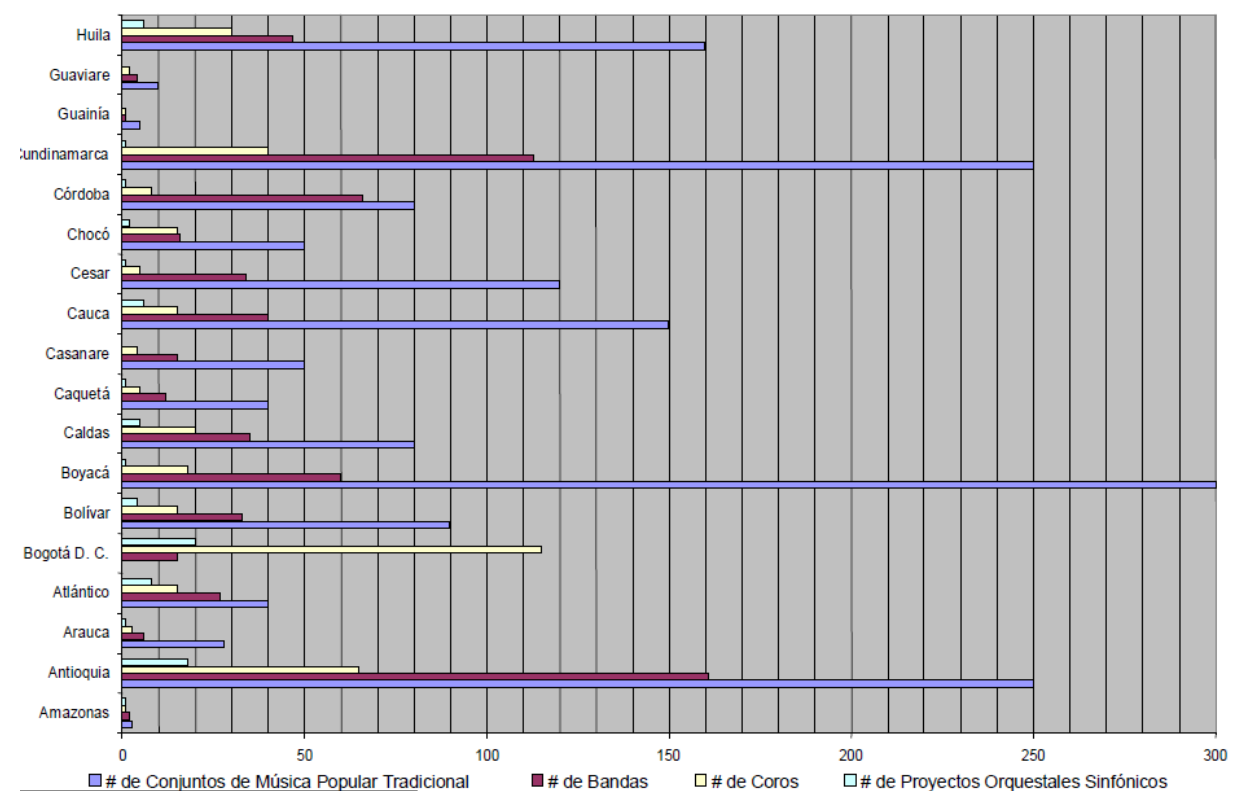

Figura 1. Ubicación de prácticas musicales de Conjuntos de Música Popular Tradicional, Bandas, Coros y Proyectos Orquestales Sinfónicos por Departamento. Fuente: Tomado de PNMC, 2004. p.20.

Las razones que justifican que el PNMC escoja los Conjuntos de Música Popular Tradicional en su proyecto formativo, es que estos obedecen a una necesidad de Contexto, pues como puede verse en la Figura 1, son el tipo de agrupación con mayor existencia en los distintos departamentos del país a penas seguido por las Bandas, lo cual permite alcanzar el elemento Cobertura respecto a la formación musical -tan buscado en la política pública- y enlaza este tipo de agrupación con lo que el PNMC llama Diálogo de Saberes, que es no solo la interacción del saber musical popular con la academia, sino cómo el saber tradicional se convierte en un insumo para la música y músicos académicos. Ante el interés utilitario que tiene el Estado sobre la Música, a su vez, el PNMC tiene una visión utilitaria de subordinación de la Música Tradicional Colombiana - o Músicas Colombianas- ante la llamada Música Académica o Clásica.

El Conservatorio del Tolima encuentra una oportunidad laboral para sus futuros graduados de Licenciatura en Música y diseña su currículo ante las necesidades manifiestas del Estado incluyendo los cuatro tipos de agrupaciones que selecciona el PNMC en sus áreas de énfasis, y por tal da ingreso a las Músicas Colombianas de manera estructural. Vemos pues, que la decisión de ingreso de estas músicas proviene del seguimiento de una política de estado más que de una deliberación y discusión interna sobre la importancia de las mismas.

(c) BORIS ALFONSO SALINAS ARIAS. THE CONTENT OF THIS ARTICLE IS THE SOLE RESPONSIBILITY OF THE AUTHORS. THE REVISTA ELECTRÓNICA DE LEEME AND UNIVERSITAT DE VALĖNCIAARE NOT LABBEFORANY LGA ACTIONSTHAT MAYARISE INVOLVING THEARTICLE'SCONTENT REVISTA ELECTRONICADELEEME - LISTA ELECTRONICA EUROPEADEMÚSICA EN LA EDUCACIÓN-HTTP://MUSICA.REDIRIS.ES.ISSN: 1575-9563 EDITORES: UNIVERSIDAD DE VALENCIA Y JESÚS TEJADA GIMÉNEZ. VISIBILIDAD DE ESTA REVISTA: EBSCO, CINDOC (CESIC), CITEFACTOR, COPAC, DIALNET, DICE (CSIC), DOAJ, E-REVISTAS (CSIC), EBSCO PREMIER, ERIH+, GALECENGAGELEARNING, IN-RECS, IRESIE, LATINDEX, MIAR, OCLCWORLDCAT, RESH, REDIB, RILMCOREJOURNALS, SUDOC, ULRICHS, ESTA REVISTA ESTÁ PUBLICADA CON EL APOYO INSTITUCIONAL DE REDIRIS-CONSEJO SUPERIOR DE INVESTIGACIONES CIENTIFICAS Y ES DE ACCESO LIBRE.CREATIVECOMMONSLICENSE 4.O BY 


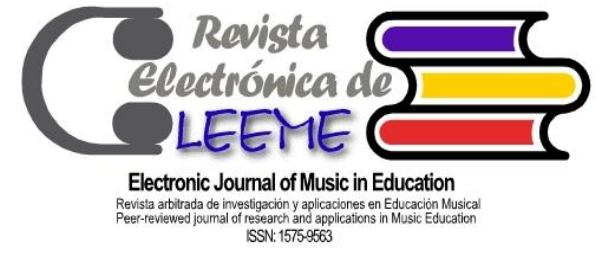

Tabla 1. Comparación Músicas Colectivas del PNMC y Áreas de énfasis de Licenciatura en Música. Figura realizada con la información disponible del PNMC (2004) y el PEP de Licenciatura en música del Conservatorio del Tolima

(2010).

\begin{tabular}{|l|l|}
\hline $\begin{array}{l}\text { Prácticas musicales Colectivas escogidas por el } \\
\text { PNMC }\end{array}$ & $\begin{array}{l}\text { Áreas de énfasis del programa Licenciatura en } \\
\text { Música }\end{array}$ \\
\hline Conjuntos de Música Popular Tradicional & $\begin{array}{l}\text { Lic. en Música con énfasis en Música Tradicional } \\
\text { Colombiana }\end{array}$ \\
\hline Las prácticas de Bandas -Banda Sinfónica- & Lic. en Música con énfasis en Vientos y Percusión \\
\hline Los Coros & Lic. en Música con énfasis en Coro \\
\hline Orquestas - de música Clásica- & Lic. en Música con énfasis en Cuerdas Frotadas \\
\hline
\end{tabular}

\section{Discusión}

Según Miñana (2006), con la entrada del Neoliberalismo a Colombia, el Arte y la Cultura comienza a verse como un negocio, donde prima la austeridad por parte del Estado y la búsqueda de diferentes ingresos privados para el sector. Las personas deben estar dispuestas a pagar para acceder al Arte pues éste se considera como producto y bien de valor más no como un derecho:

"En la última década hemos asistido en Colombia... a una situación paradójica: mientras se promulgaban modernas y participativas leyes de cultura (1997 en el caso colombiano), acordes con nuevas y más democráticas constituciones (1991 en Colombia), se producía un recorte significativo en los recursos públicos destinados al área de cultura, artes y patrimonio.

Mientras que en 1997 el presupuesto del Ministerio de Cultura fue de 107.749 millones de pesos, el presupuesto 2006 fue de 84.206 (ambas cifras en pesos constantes de 2002, sin considerar la inflación). En este periodo hubo años en la que la inversión bajó a 49.199 (año 2000). El Ministerio y Planeación Nacional son conscientes de los precarios recursos que se asignan al sector. Pero, en lugar de incrementarlos proponen: "Dada la escasez de recursos en el sector es indispensable que estos se utilicen con la mayor eficiencia y transparencia posible" (Colombia 2002:28) y "Como complemento a los recursos propios, $\mathrm{MC}$ debe conseguir una mayor cantidad de recursos externos que le permitan financiar sus programas de acuerdo con los lineamientos de la política cultural" (Miñana 2006, p.1).

La reducción de presupuesto para la Cultura dada por la base ideológica Neoliberal en la que entró el país desde el Gobierno de Gaviria (1990 - 1994) con la Apertura Económica, marca un cambio de visión, la cultura pasó a ser valorada por su utilidad en "proyectos de convivencia, de resolución de conflictos, de formación para la paz orientadas a esas inmensas juventudes marginalizadas" (Miñana 2006, p.4). La Cultura -incluyendo en esta a todas las manifestaciones artísticas- pasó a tener un valor instrumental dada su pertinente utilidad de pacificación en un país con más de medio siglo de Conflicto Armado, como lo deja ver el Documento CONPES 3162 de 2002:

"Este documento presenta a consideración del Consejo Nacional de Política Económica y Social (CONPES) los lineamientos de política orientados a la sostenibilidad del Plan Nacional de Cultura 


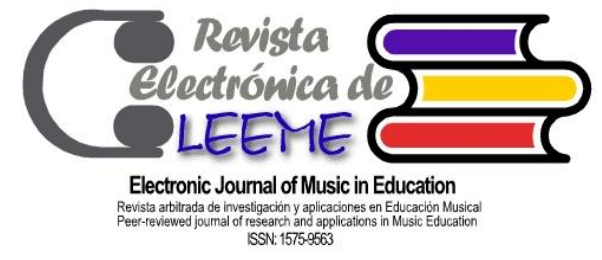

BORIS ALFONSO SALINAS ARIAS
EL INGRESO DE LAS MÚSICAS COLOMBIANAS A LA EDUCACIÓN SUPERIOR DEL CONSERVATORIO DEL TOLIMA (REVISTAELECTRÓNICA DE LEEME) NÚMERO 41, PP. 1-15 HTTPS://OJS.UV.ES/INDEX.PHP/LEEME/INDEX

2001 - 2010. Se trata de un esfuerzo orientado a fortalecer tanto al Ministerio de Cultura como los procesos del sector, con el fin de aprovechar el potencial que tiene la cultura para fomentar los valores, la creatividad, la cohesión social, el mejoramiento de la calidad de vida y la búsqueda de la paz" (CONPES 3162 de 2002, p.2).

Para el año 2002 la Música fue escogida como práctica artística insignia en el proceso de pacificación -y fue la única práctica artística con un plan propio durante casi una década -. Este rumbo aparece trazado en el punto 48 del Manifiesto Democrático del entonces candidato y posterior presidente Álvaro Uribe Vélez -2002 a 2008- "Cuando un niño abraza un instrumento musical, jamás empuñará un fusil contra el prójimo” (Uribe, 2002, p.7).

En este mismo año -2002- nace el Plan Nacional De Música Para La Convivencia (PNMC), el cual tuvo una fuerte relación con el Plan Colombia, política de cooperación y lucha contra las drogas entre Estados Unidos y Colombia que comenzó con el Gobierno de Andrés Pastrana (1998 - 2002), que a través del CONPES 3134 de 2001 Plan Colombia: Infraestructura Social Y Gestión Comunitaria, fijó como objetivo "apoyar a los municipios más pobres y afectados por la violencia a través de acciones en tres áreas" $(2001$, p.2) primero construir y mejorar la infraestructura social básica para luego realizar allí programas sociales y culturales con entidades nacionales donde se vincula directamente al Ministerio de Cultura. Recursos provenientes del Plan Colombia llegaron al Plan Nacional de Música para la Convivencia, el cual concibe a la música así:

\footnotetext{
"La música es asumida en el PNMC desde su naturaleza intrínseca y su fuerza propia y no solamente como un instrumento de pacificación y cohesión social. Si bien es cierto que la música y las demás manifestaciones artísticas por sí mismas no contrarrestan las expresiones violentas del conflicto, sí posibilitan un mejor desarrollo perceptivo, cognoscitivo y emocional de los individuos y contribuye a fortalecer valores sociales.

El hecho de que el fenómeno musical tenga la facultad de conmovernos, esto es, de dar forma y expresión a nuestros mundos afectivos, lo ubica como una alternativa de vital importancia en la construcción de tejidos sociales" (PNMC, 2004, p.3).
}

Con la Ley 812 de 2003 se aprobó el Plan de Desarrollo Nacional de Colombia en cuyo articulado quedó el PNMC no en la sección cultural sino en el capítulo II que habla de la Seguridad Democrática, en medio de los temas de fortalecimiento de la convivencia y los valores, fortalecimiento de las fuerzas armadas, lucha contra las drogas y la subversión.

Más allá de si la música puede o no generar hábitos de convivencia o una cultura de Paz, lo cierto es que el Estado se inclinó sobre esta propiedad o facultad de la música más que sobre el arte mismo. El Plan Nacional de Música fue uno de los pocos programas sociales que financió el Plan Colombia, con la finalidad de generar Convivencia; música utilizada como forma de pacificación a través de la creación de tejido social, que con organizaciones como la Fundación Nacional Batuta pudo ver cómo en regiones azotadas por el Conflicto Armado como el municipio de Planadas, sur del Tolima, se creaban -y actualmente existen- agrupaciones y escuelas de música

(c) BORIS ALFONSO SALINAS ARIAS. THE CONTENT OF THIS ARTICLE IS THE SOLE RESPONSIBILITY OF THE AUTHORS. THE REVISTA ELECTRÓNICA DE LEEME AND UNIVERSITAT DE VALĖNCIA ARE NOT LIABLE FOR ANY LEGAL ACTIONS THAT MAYARISE INVOLVING THE ARTICLE'S CONTENT. REVISTA ELECTRÓNICA DE LEEME -LISTA ELECTRÓNICA EUROPEA DE MÚSICA EN LA EDUCACIÓN-HTTP://MUSICA.REDIRIS.ES.ISSN: 1575-9563 EDITORES: UNIVERSIDAD DE VALENCIA Y JESÚS TEJADA GIMÉNEZ. VISIBILIDAD DE ESTA REVISTA: EBSCO, CINDOC (CESIC), CITEFACTOR, COPAC, DIALNET, DICE (CSIC), DOAJ, EREVISTAS (CSIC), EBSCO PREMIER, ERIH+, GALECENGAGELEARNING, IN-RECS, IRESIE, LATINDEX, MIAR, OCLCWORLDCAT, RESH, REDIB, RILMCOREJOURNALS, SUDOC, ULRICHS, ESTA REVISTA
CIENTIFICAS Y ES DE ACCESO LIBRE.CREATIVECOMMONSLICENSE 4.O BY 


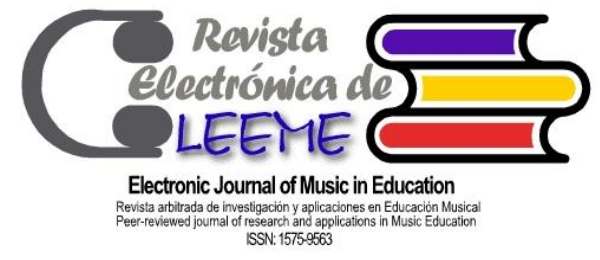

EL INGRESO DE LAS MÚSICAS COLOMBIANAS BORIS ALFONSO SALINAS ARIAS CONSERVATORIO DEL TOLIMA (REVISTAELECTRÓNICA DE LEEME) NÚMERO 41, PP. 1-15 HTTPS://OJS.UV.ES/INDEX.PHP/LEEME/INDEX

que pudieran prevenir el reclutamiento armado de menores de edad y dar atención social a víctimas del Conflicto. En este momento programas como Música para la Reconciliación, financiados por el Departamento de la Prosperidad Social (DPS) son llevados a cabo para este tipo de población.

Para el año 2006 se da un segundo impulso al PNMC a través del CONPES 3409 Lineamientos para el fortalecimiento del Plan Nacional del Música para la Convivencia, esto ocurría en momentos álgidos del Conflicto Armado Colombiano y la política de Seguridad Democrática del gobierno Uribe:

"Este documento presenta a consideración del Consejo Nacional de Política Económica y Social una propuesta para avanzar en la consolidación del Plan Nacional de Música para la ConvivenciaPNMC. Se trata de un esfuerzo orientado a fortalecer los procesos del subsector de la música, las agendas intersectoriales y el presupuesto del PNMC, con el fin de aprovechar el potencial que tiene la música para fomentar los valores, la creatividad, la cohesión social, el mejoramiento de la calidad de vida y la búsqueda de la convivencia pacífica" (CONPES 3409. 2006, p.2).

Nos encontramos en un escenario en que la música fue concebida como elemento de pacificación, pero no es la primera vez que algo similar ocurre, recordemos que Descartes con su Tratado de las Pasiones del Alma (1649) influyó en lo que se conoció durante el Barroco como la Teoría de los Afectos que buscaba causar y controlar las emociones humanas a partir del arte, algo que en la Antigua Grecia se estudiaba y se conocía como el Ethos. En Colombia, "Niños y jóvenes guerrilleros y paramilitares abrazan sus guitarras en el descanso de la batalla, y ayer la infancia y las juventudes nazis entonaban himnos patrióticos en honor al führer." (Miñana 2006, p.6).

El asunto de todo esto es el uso de la Música para pacificar a una población, el arte aparece como un producto por el cual se debe pagar, hay una reducción de los recursos económicos por parte del Estado para atender culturalmente a la Nación, el gobierno suprime programas como la Orquesta Sinfónica de Colombia y la Banda Nacional (Decreto 3210 de 2002), el grueso de la población queda sin acceso a la formación musical y cultural focalizando la atención en la población que vive en los puntos clave del conflicto armado.

Al ser la Música asumida por el Estado como una forma de combatir en el conflicto, es predecible que los artistas y organizaciones como el Conservatorio del Tolima deban cambiar su concepción y discurso de la Música por la Música, por expresión humana, por búsqueda de belleza, a una concepción y discurso de la Música en pro de la Convivencia, al fin y al cabo, en Colombia la gran mayoría de Artistas y ONG así como los Gobiernos locales, obtienen importantes recursos económicos del Estado Central.

Ahora, ¿Cuál es la posición ideológica del Conservatorio del Tolima frente a las Músicas Colombianas y la política de pacificación del Estado? El Conservatorio fijó una posición utilitaria sobre la música muy parecida a la visión instrumental que posee el Estado sobre la misma, por el

(c) BORIS ALFONSO SALINAS ARIAS. THE CONTENT OF THIS ARTICLE IS THE SOLE RESPONSIBILITY OF THE AUTHORS. THE REVISTA ELECTRÓNICA DE LEEME AND UNIVERSITAT DE VALÈNCIA ARE NOT LIABLE FOR ANY LEGAL ACTIONS THAT MAYARISE INVOLVING THE ARTICLE'S CONTENT. REVISTA ELECTRÓNICA DE LEEME - LISTA ELECTRÓNICA EUROPEA DE MÚSICA EN LA EDUCACIÓN-HTTP://MUSICA.REDIRIS.ES.ISSN: 1575-9563 EDITORES: UNIVERSIDAD DE VALENCIA Y JESÚS TEJADA GIMÉNEZ. VISIBILIDAD DE ESTA REVISTA: EBSCO, CINDOC (CESIC), CITEFACTOR, COPAC, DIALNET, DICE (CSIC), DOAJ, E-REVISTAS (CSIC), EBSCO PREMIER, ERIH+, GALECENGAGELEARNING, IN-RECS, IRESIE, LATINDEX, MIAR, OCLCWORLDCAT, RESH, REDIB, RILMCOREJOURNALS, SUDOC, ULRICHS, ESTA REVISTA ESTÁ PUBLICADA CON EL APOYO INSTITUCIONAL DE REDIRIS-CONSEJO SUPERIOR DE INVESTIGACIONES CIENTIFICAS Y ES DE ACCESO LIBRE.CREATIVECOMMONSLICENSE 4.0 BY 


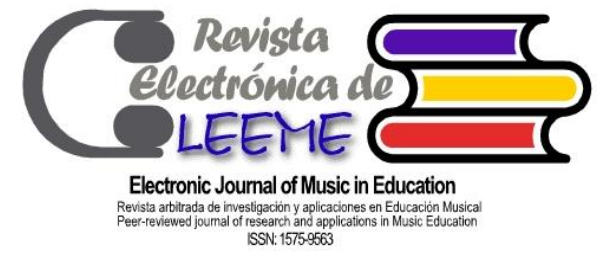

EL INGRESO DE LAS MÚSICAS COLOMBIANAS BORIS ALFONSO SALINAS ARIAS CONSERVATORIO DEL TOLIMA

(REVISTAELECTRÓNICA DE LEEME) NÚMERO 41, PP. 1-15 $\frac{\text { HTTPS://OJS.UV.ES/INDEX.PHP/LEEME/INDEX }}{\text { DOI: } 10.7203 / \text { LEEME.41.10220 }}$

cual se justifica la música a partir de los otros beneficios que ésta puede llegar a brindar, mas no por su valor y desarrollo propio:

"En nuestra institución se considera la práctica musical en la escuela como el medio que permite el desarrollo de otras competencias cognitivas y afectivas que dan una integralidad al estudiante y lo preparan para la vida en comunidad" (PEP. 2010, p.9).

Esta posición es completamente coherente con uno de los objetivos principales que la institución se impuso, pues según el PEI (2015) el Conservatorio del Tolima se propone "liderar programas educativos musicales que favorezcan la convivencia y la construcción pacifica de nuestro país" (p.11).

En el PEI (2015) del Conservatorio del Tolima las Músicas Colombianas aparecen como algo menor, pues en sus ocho Objetivos institucionales no se propone el estudio de estas, al contrario, la Música Clásica sí es parte fundamental de su misión, en el Objetivo 3, el Conservatorio del Tolima se propone "Ser fuente de investigación, interpretación y difusión de la música erudita nacional e internacional" (p.11, 2015). Cabe aclarar que las Músicas Colombianas no hacen parte de lo que el Conservatorio llama música erudita, pues esta es otra forma de llamar a la Música Clásica o de Europa Central.

En las páginas 6 y 7 del PEI (2015), el Conservatorio relata la existencia de nueve agrupaciones de las cuales ocho son de música Clásica. A continuación, se presenta un extracto de estas páginas:

\section{"AGRUPACIONES ARTÍSTICAS EXISTENTES \\ ORQUESTA PRE-INFANTIL}

Conformada por niños(as) entre 6 y 10 años de edad, estudiantes de la Escuela de Música. Inició en el año 2002. Su repertorio está constituido por obras clásicas y populares del ámbito regional, nacional e internacional.

(...)

\section{ORQUESTA SINFÓNICA DEL CONSERVATORIO DEL TOLIMA}

Inició si actividad en el año 1906. Es la agrupación sinfónica de mayor nivel, está conformada por docentes y estudiantes. Ofrece conciertos periódicos en el Salón Alberto Castilla y participa en los eventos especiales que organiza el Conservatorio del Tolima en la ciudad y fuera de ella.

ENSAMBLES Y OTROS GRUPOS

La actividad musical de la institución permite que se creen y organicen otros grupos musicales para permitir expresar la creatividad, la innovación y la libre expresión musical de profesores y estudiantes. Por esta razón se han organizado agrupaciones como el Grupo de Jazz, la Estudiantina, grupo de percusión folclórica, grupos de rock y grupos de música folclórica" (PEI, 2015, p.6-7).

Es de observar que mientras las agrupaciones de Música Clásica fueron enunciadas una por una, descritas incluso con año de creación y repertorio a interpretar, a diferencia, las agrupaciones de música no Clásica como Colombiana o Popular se acuñaron en una única categoría -Ensambles y otros grupos- ya que la institución permite la existencia de otras agrupaciones para dar un espacio expresión de estudiantes y profesores, es decir, no son 


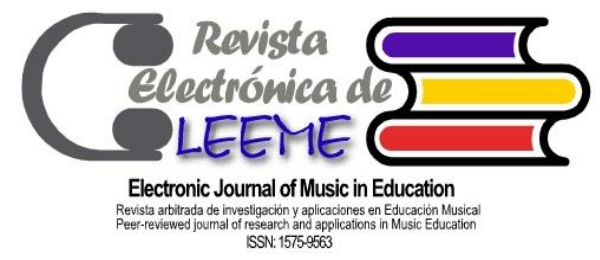

EL INGRESO DE LAS MÚSICAS COLOMBIANAS BORIS ALFONSO SALINAS ARIAS CONSERVATORIO DEL TOLIMA (REVISTAELECTRÓNICA DE LEEME) NÚMERO 41, PP. 1-15 $\frac{\text { HTTPS://OJS.UV.ES/INDEX.PHP/LEEME/INDEX }}{\text { DOI: } 10.7203 / \text { LEEME.41.10220 }}$

agrupaciones que hacen parte de las prioridades, no son objeto de estudio ni parte fundamental de la identidad institucional, sino manifestaciones artísticas de algunos estudiantes y docentes, toleradas por la universidad. Aun así, resulta apreciable cómo dentro de esos otros grupos dos fueron escritos con mayúscula inicial, el grupo de Jazz y la Estudiantina, lo que representa una hegemonía interna en la que los grupos de Folclor escritos en letras minúsculas sugieren una categoría inferior.

Es comprensible la preocupación del Conservatorio por la posibilidad laboral de sus graduados, pero es cuestionable que la inserción de las Músicas Colombianas no se deba a un proceso de reflexión, análisis y crítica sobre estas sino por la implementación a ciegas del modelo de Estado planteado por Gobiernos Neoliberales comprometidos con el Plan Colombia que construyeron un Plan de Música para la pacificación. A su vez, se pudo develar a lo largo del estudio que las Músicas Colombianas son asumidas con un interés curricular de tipo técnico.

\section{Conclusiones}

La Universidad no debe renunciar a su autonomía de pensar e idear un país y reflexionar cuál debe ser su aporte en la construcción de nación, criticar y enriquecer la política pública o buscar nuevas alternativas ante los cambios que el pensamiento Neoliberal ha traído al Estado sobre las artes y el oficio del Músico.

La razón de ser de la Universidad no puede estar atada al Estado y gobierno de turno, pues su deber es con el saber científico y con la Humanidad, su Misión tiene que estar por encima de la verdad política. La Universidad debe ser la fuente de cambios y mejoras de una sociedad. Se necesita una universidad más propositiva y menos responsorial, esto es, que proponga cambios a la sociedad desde una reflexión profunda y no que se limite a responder a las necesidades momentáneas que el estado y el mercado pide.

Frente al postconflicto el Licenciado en Música parece estar más que preparado a las necesidades del estado y su PNMC; pero, si para el año 2020 el PNMC desaparece o cambia, ¿Estarán los Licenciados en Música preparados para los cambios laborales que plantee el sector? ¿Continuarán las Músicas Colombianas siendo parte del currículo del programa Licenciatura en Música o regresarán a ser parte de lo prohibido?, en este sentido hay que decir que la Universidad debe tener un compromiso permanente con los distintos saberes y el estudiar las músicas nacionales no debería ser iniciativa de intereses trasnacionales.

Si bien el PNMC surge como iniciativa para la pacificación del país, la finalidad del Plan Colombia -su financiador- no estuvo encaminada a la solución del conflicto armado colombiano, sino que obedeció a la política exterior de seguridad de Estados Unidos en el marco de la de guerra contra las drogas. Colombia fue visto como estado fallido, luego del posicionamiento del tráfico

(c) BORIS ALFONSO SALINAS ARIAS. THE CONTENT OF THIS ARTICLE IS THE SOLE RESPONSIBILITY OF THE AUTHORS. THE REVISTA ELECTRÓNICA DE LEEME AND UNIVERSITAT DE VALĖNCIA ARE NOT LIABLE FOR ANY LEGAL ACTIONS THAT MAYARISE INVOLVING THE ARTICLE'S CONTENT. REVISTA ELECTRÓNICA DE LEEME -LISTA ELECTRÓNICA EUROPEA DE MÚSICA EN LA EDUCACIÓN-HTTP://MUSICA.REDIRIS.ES.ISSN: 1575-9563 EDITORES: UNIVERSIDAD DE VALENCIA Y JESÚS TEJADA GIMÉNEZ. VISIBILIDAD DE ESTA REVISTA: EBSCO, CINDOC (CESIC), CITEFACTOR, COPAC, DIALNET, DICE (CSIC), DOAJ, EREVISTAS (CSIC), EBSCO PREMIER, ERIH+, GALECENGAGELEARNING, IN-RECS, IRESIE, LATINDEX, MIAR, OCLCWORLDCAT, RESH, REDIB, RILMCOREJOURNALS, SUDOC, ULRICHS, ESTA REVISTA
CIENTIFICAS Y ES DE ACCESO LIBRE.CREATIVECOMMONSLICENSE 4.0 BY 


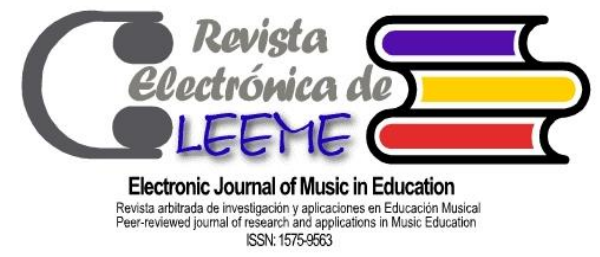

BORIS ALFONSO SALINAS ARIAS
EL INGRESO DE LAS MÚSICAS COLOMBIANAS ALA EDUCACIÓN SUPERIOR DDLL CONSERVATORIO DEL TOLIMA (REVISTAELECTRÓNICA DE LEEME) NÚMERO 41, PP. 1-15 HTTPS://OJS.UV.ES/INDEX.PHP/LEEME/INDEX

de drogas en la economía de las guerrillas y la estrecha relación entre las fuerzas políticas colombianas con el narcotráfico, lo que hizo percibir a este país como un inminente narco estado (Monroy, 2015).

El objetivo del Plan Colombia fue lucha contra el terrorismo y las drogas pero también obedeció a otras lógicas de Colonialidad y mercado; actualmente se sabe que este tuvo directa incidencia en la decisión del presidente Pastrana (1998-2002) en realizar los diálogos de paz con las FARC, así como en la negociación del TLC entre Estados Unidos y Colombia en el gobierno de Uribe (2002-2008):

"El plan Colombia no es una mera repetición de las pretensiones imperialistas del pasado, sino que hace parte de un diseño global mucho más complejo en el cual se combinan elementos expansionistas y militaristas con fuertes distancias aislacionistas... la "anexión" de América Latina al modelo hegemónico de producción ya no se realiza bajo la forma del capitalismo liberal clásico. Difiere, entre otras cosas, en que opera sin necesidad de apelar al "contrato social" y se concentra en la expropiación ya no solo de la mano de obra barata, sino de conocimientos subalternos que antes se tenían por "premodernos"... Es decir que la incorporación de las periferias a la economía del sistema mundo continúa pasando por la "vía colonial" - la colonialidad del poder de la que habla Aníbal Quijano (1992)- pero ahora asumiendo formas diferentes. Todo esto significa que las luchas frente al modelo hegemónico de globalización pasan, en parte, por la afirmación de formas locales de conocimiento... pues éstas ya se encuentran integradas a redes globales" (Castro-Gómez \& Guardiola, 2002, p.70-71).

Hay una estrecha e inquietante relación entre el Plan Colombia, los TLC, la legislación colombiana sobre los derechos de propiedad intelectual, el Mercado mundial y el Neoliberalismo, con el interés asumido por el PNMC hacia los saberes musicales, las Músicas de Colombia. En el tratado de Libre Comercio Estados Unidos aceptó reconocer los conocimientos tradicionales y el folclor colombiano como conocimientos especiales en el marco del acuerdo de la ADPIC, solo a cambio de que Colombia incluyera en el TLC el patentamiento de vegetales y animales, junto al tratado UPOV respecto a derechos de autor, así como de interpretación de fonogramas, entre otros (Hernández, 2015).

En este aspecto queda decir que la capacidad científica, económica y tecnológica de Colombia frente a Estados Unidos es completamente desequilibrada. Colombia tiene el perfil para seguir siendo un productor de materias primas con la diferencia que ahora puede abastecer el comercio de patentes de Estados Unidos.

Insertar las Músicas Colombianas así como los saberes ancestrales en el Mercado global es una tarea asumida por los tratados firmados por el Estado colombiano, que debe ser analizada con detenimiento por los artistas, la academia y los diversos miembros pluriétnicos y multiculturales del país, más aun con la fuerte política extraccionista y minera que los últimos cinco gobiernos han profesado, en el que las obras musicales de carácter colectivo que pertenecen a las diversas etnias del país no cuentan con propiedad intelectual.

(C) BORIS ALFONSO SALINAS ARIAS. THE CONTENT OF THIS ARTICLE IS THE SOLE RESPONSIBILITY OF THE AUTHORS. THE REVISTA ELECTRÓNICA DE LEEME AND UNIVERSITAT DE VALĖNCIA ARE NOT LIABLE FOR ANY LEGAL ACTIONS THAT MAYARISE INVOLVING THE ARTICLE'S CONTENT. REVISTA ELECTRÓNICA DE LEEME -LISTA ELECTRÓNICA EUROPEA DE MÚSICA EN LA EDUCACIÓN-HTTP://MUSICA.REDIRIS.ES.ISSN: 1575-9563 EDITORES: UNIVERSIDAD DE VALENCIA Y JESÚS TEJADA GIMÉNEZ. VISIBILIDAD DE ESTA REVISTA: EBSCO, CINDOC RESH, REDIB, RILMCOREJOURNALS, SUDOC, ULRICHS, ESTA REVISTA ESTA PUBLICADA CON EL APOYO INSTITUCIONAL DE REDIRIS-CONSEJO SUPERIOR DE INVESTIGACIONES CIENTIFICAS Y ES DE ACCESO LIBRE.CREATIVECOMMONSLICENSE 4.0 BY 


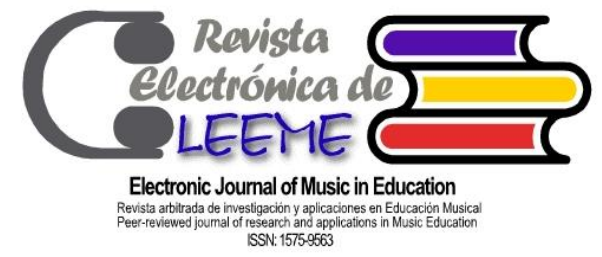

EL INGRESO DE LAS MÚSICAS COLOMBIANAS BORIS ALFONSO SALINAS ARIAS CONSERVATORIO DERIOR DEL (REVISTAELECTRÓNICA DE LEEME) NÚMERO 41, PP. 1-15 HTTPS://OJS.UV.ES/INDEX.PHP/LEEME/INDEX

\section{Referencias}

Camacho, F., Díaz, L. y Pérez, A. (2000). "El papel de la música popular en la Facultad de Educación y Artes del Conservatorio del Tolima”. En J. González (Presidencia), III Congreso Latinoamericano de la Asociación Internacional para el Estudio de la Música Popular (IASPM$A L)$. Bogotá, Colombia. Recuperado de http://iaspmal.com/index.php/2016/03/02/actas-iiicongreso-bogota-colombia-2000/

Castro-Gómez, S. y Guardiola, O. (2002). "El Plan Colombia o de cómo una historia local se convierte en diseño Global”. En C. Walsh, F. Schiwy, S. Castro-Gómez (Ed), Indisciplinar las ciencias sociales: Geopolíticas del conocimiento y colonialidad del poder. Perspectivas desde lo andino. (pp. 61-71). Quito: Universidad Andina Simón Bolivar y Editorial Abya Yala.

Congreso de la República de Colombia (1994). Ley 112 de 1994. Recuperado de http://www.secretariasenado.gov.co/senado/basedoc/ley_0112_1994.html

Consejo Nacional de Política Económica y Social de la República de Colombia (2001). Documento CONPES 3134. Plan Colombia: Infraestructura Social y Gestión Comunitaria. Recuperado de https://colaboracion.dnp.gov.co/CDT/CONPES/Econ\%C3\%B3micos/3134.pdf

Consejo Nacional de Política Económica y Social de la República de Colombia (2002). Documento CONPES 3162. Lineamientos para la sostenibilidad del plan Nacional de Cultura 2001-2010 "Hacia una ciudadanía democrática Cultural". Recuperado de http://www.nuevalegislacion.com/files/susc/cdj/conc/conpes_3162.pdf

Consejo Nacional de Política Económica y Social de la República de Colombia (2002). Documento CONPES 3191. Fortalecimiento del Programa Nacional de Bandas de Vientos. Recuperado de https://colaboracion.dnp.gov.co/CDT/CONPES/Econ\%C3\%B3micos/3191.pdf

Consejo Nacional de Política Económica y Social de la República de Colombia (2006). Documento CONPES 3409. Lineamientos para el fortalecimiento del Plan Nacional del Música para la Convivencia. Recuperado de http://www.icbf.gov.co/cargues/avance/docs/conpes_dnp_3409_2006.htm

Conservatorio del Tolima (2006). Facultad de Educación y Artes, programas Académicos, años 1997 - 2006. Legajos 1 al 19. Ibagué, Colombia: Fondo Documental Conservatorio del Tolima.

Conservatorio del Tolima (2015). Plan de Curso por Competencias, programa Licenciatura en Música, plan 2012. Ibagué, Colombia: Facultad de Educación y Artes del Conservatorio del Tolima.

(c) BORIS ALFONSO SALINAS ARIAS. THE CONTENT OF THIS ARTICLE IS THE SOLE RESPONSIBILITY OF THE AUTHORS. THE REVISTA ELECTRÓNICA DE LEEME AND UNIVERSITAT DE VALĖNCIA ARE NOT LIABLE FOR ANY LEGAL ACTIONS THAT MAYARISE INVOLVING THE ARTICLE'S CONTENT. REVISTA ELECTRÓNICA DE LEEME -LISTA ELECTRÓNICA EUROPEA DE MÚSICA EN LA EDUCACIÓN-HTTP://MUSICA.REDIRIS.ES.ISSN: 1575-9563 EDITORES: UNIVERSIDAD DE VALENCIA Y JESÚS TEJADA GIMÉNEZ. VISIBILIDAD DE ESTA REVISTA: EBSCO, CINDOC (CESIC), CITEFACTOR, COPAC, DIALNET, DICE (CSIC), DOAJ, EREVISTAS (CSIC), EBSCO PREMIER, ERIH+, GALECENGAGELEARNING, IN-RECS, IRESIE, LATINDEX, MIAR, OCLCWORLDCAT, RESH, REDIB, RILMCOREJOURNALS, SUDOC, ULRICHS, ESTA REVISTA ESTÁ PUBLICADA CON EL APOYO INSTITUCIONAL DE REDIRISCCONSEJO SUPERIOR DE INVESTIGACIONES CIENTIFICAS Y ES DE ACCESO LIBRE.CREATIVECOMMONSLICENSE 4.O BY 


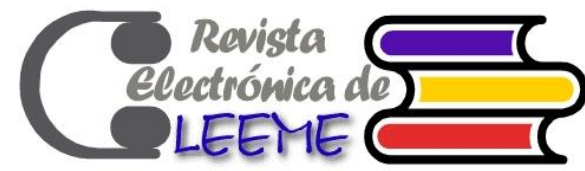

Electronic Journal of Music in Education

Revista artitrada de investigación y aplicacioneses en Educación Musica

ISSN: 1575.9563

Conservatorio del Tolima (2015). Proyecto Educativo Institucional (PEI). Ibagué, Colombia: Facultad de Educación y Artes, Conservatorio del Tolima.

Conservatorio del Tolima (2010). PEP: Proyecto Educativo del Programa de Licenciatura en Música: informe para la Renovación del Registro Calificado. Ibagué, Colombia: Facultad de Educación y Artes, Conservatorio del Tolima.

Hernández, R. (2015). Tratado de libre comercio Colombia-EEUU, propiedad intelectual y conocimientos tradicionales en el contexto de los Derechos de las Comunidades Afrocolombianas, Negra, Raizal y Palenquera. Revista Virtual de Ciencias Sociales y Humanas PSICOESPACIOS, 9 (15), 283-327.

Mignolo, W. (2002). "Las Geopolíticas de Conocimiento y Colonialidad del Poder". En C. Walsh, F. Schiwy, S. Castro-Gómez (Ed.), Indisciplinar las ciencias sociales: Geopolíticas del conocimiento y colonialidad del poder. Perspectivas desde lo andino. (pp. 17-42). Quito: Universidad Andina Simón Bolivar y Editorial Abya Yala.

Ministerio de Cultura. (2004). Plan Nacional de Música para la Convivencia. Bogotá: Ministerio de Cultura.

Ministerio de Cultura. (2012). Plan Nacional del Música para la Convivencia, Guía para Alcaldes y Gobernadores de Colombia. Bogotá: Imprenta Nacional.

Ministerio de Educación Nacional (2008). Orientaciones Pedagógicas para la Educación Artística y Cultural: Educación Preescolar, Básica y Media. Bogotá: Ministerio de Educación Nacional.

Ministerio de Educación Nacional (2011). Articulación de la Educación con el Mundo Productivo. Bogotá: Imprenta Nacional de Colombia.

Miñana, C. Ariza y A. Arango, C. (2006). "Formación Artística y Cultural: ¿arte para la convivencia?". En M. Giraldo (Presidencia), VII Encuentro para la promoción y difusión del patrimonio inmaterial en países iberoamericanos. Caracas, Venezuela.

Monroy, M. (2015). Acontecimientos históricos de la política exterior de Estados Unidos hacia Colombia antes del Plan Colombia 1998. Revista Científica General José María Córdova, 13 (15), 19-61.

Páramo, P. (2013). La Investigación en Ciencias Sociales: estrategias de investigación. Bogotá: Editorial Universidad Piloto de Colombia.

Quijano, A. (2000). "Colonialidad del Poder, eurocentrismo y América Latina”. En E. Lander. (Ed.), La colonialidad del saber: eurocentrismo y ciencias sociales. Perspectivas

(C) BORIS ALFONSO SALINAS ARIAS. THE CONTENT OF THIS ARTICLE IS THE SOLE RESPONSIBILITY OF THE AUTHORS. THE REVISTA ELECTRÓNICA DE LEEME AND UNIVERSITAT DE VALĖNCIA ARE NOT LIABLE FOR ANY LEGAL ACTIONS THAT MAYARISE INVOLVING THE ARTICLE'S CONTENT. REVISTA ELECTRÓNICA DE LEEME -LISTA ELECTRÓNICA EUROPEA DE MÚSICA EN LA EDUCACIÓN-HTTP://MUSICA.REDIRIS.ES.ISSN: 1575-9563 EDITORES: UNIVERSIDAD DE VALENCIA Y JESÚS TEJADA GIMÉNEZ. VISIBILIDAD DE ESTA REVISTA: EBSCO, CINDOC (CESIC), CITEFACTOR, COPAC, DIALNET, DICE (CSIC), DOAJ, EREVISTAS (CSIC), EBSCO PREMIER, ERIH+, GALECENGAGELEARNING, IN-RECS, IRESIE, LATINDEX, MIAR, OCLCWORLDCAT, RESH, REDIB, RILMCOREJOURNALS, SUDOC, ULRICHS, ESTA REVISTA ESTÁ PUBLICADA CON EL APOYO INSTITUCIONAL DE REDIRIS-CONSEJO SUPERIOR DE INVESTIGACIONES CIENTIFICAS Y ES DE ACCESO LIBRE.CREATIVECOMMONSLICENSE 4.O BY 


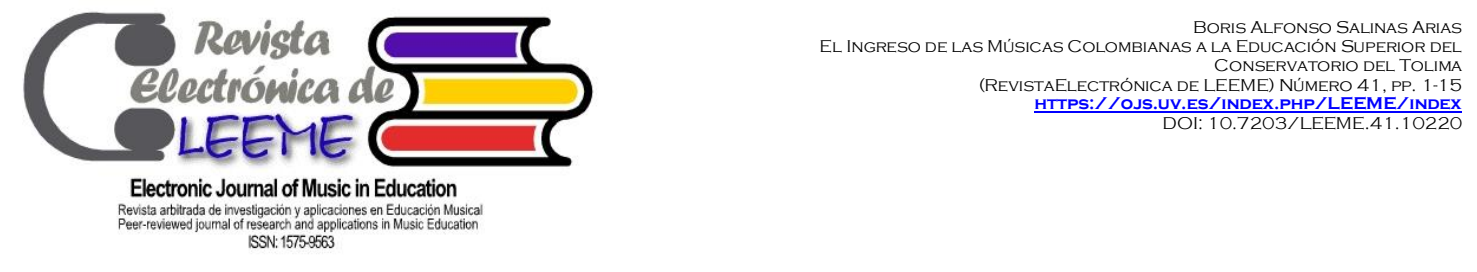

Latinoamericanas. (pp. 201-246). Buenos Aires: CLACSO. Consejo Latinoamericano de Ciencias Sociales.

Uribe, A. (2002). Manifiesto Democrático. Recuperado de http://www.mineducacion.gov.co/1621/articles-85269 archivo pdf.pdf

(c) BORIS ALFONSO SALINAS ARIAS. THE CONTENT OF THIS ARTICLE IS THE SOLE RESPONSIBILITY OF THE AUTHORS. THE REVISTA ELECTRÓNICA DE LEEME AND UNIVERSITAT DE VALĖNCIA ARE NOT LIABLE FOR ANY LEGAL ACTIONS THAT MAYARISE INVOLVING THE ARTICLE'S CONTENT. REVISTA ELECTRÓNICA DE LEEME -LISTA ELECTRÓNICA EUROPEA DE MÚSICA EN LA EDUCACIÓN-HTTP://MUSICA.REDIRIS. ES.ISSN: 1575-9563 EDITORES: UNIVERSIDAD DE VALENCIA Y JESÚS TEJADA GIMÉNEZ. VISIBILIDAD DE ESTA REVISTA: EBSCO, CINDOC (CESIC), CITEFACTOR, COPAC, DIALNET, DICE (CSIC), DOAJ, E-REVISTAS (CSIC), EBSCO PREMIER, ERIH+, GALECENGAGELEARNING, IN-RECS, IRESIE, LATINDEX, MIAR, OCLCWORLDCAT,
RESH, REDIB, RILMCOREJOURNALS, SUDOC, ULRICHS, ESTA REVISTA ESTA PUBLICADA CON EL APOYO INSTITUCIONAL DE REDIRIS-CONSEJO SUPERIOR DE INVESTIGACIONES CIENTIFICAS Y ES DE ACCESO LIBRE.CREATIVECOMMONSLICENSE 4.0 BY 